\title{
Rahmenbedingungen kommunaler Jugendpolitik: Strukturmuster und Besonderheiten der kommunalen Ebene
}

\section{Lars Holtkamp und Stephan Grohs}

\section{Einleitung}

Jugendpolitik findet in erster Linie auf kommunaler Ebene statt. Zwar stammen die wesentlichen Gesetze und Förderprogramme von Bund und Ländern, deren letztendliche Umsetzung findet jedoch bis auf wenige Ausnahmen auf der lokalen Ebene statt. Die lokalen Spielräume sind teils erheblich, zudem setzen zahlreiche Kommunen eigene Schwerpunkte in der Jugendpolitik. Die Kommunen führen also auf der einen Seite pflichtige Selbstverwaltungsaufgaben aus, deren Spektrum durch Bundes- und Landesgesetze, insbesondere das Kinder- und Jugendhilfegesetz (SGB VIII) vorgegeben werden. Hierzu gehören die Jugendhilfe und das Angebot von Tagesbetreuung. Andererseits gestalten und fördern die Kommunen im Rahmen ihrer freiwilligen Aufgaben in Zusammenarbeit mit einer Vielzahl anderer Akteure Angebote für Jugendliche, die von Jugendtreffs über Beratungsangebote bis zu Jugendfreizeiten uns Sportveranstaltungen reichen.

Zum Verständnis der Jugendpolitik ist es daher unabdingbar, die kommunalpolitischen Rahmenbedingungen, wesentliche Akteure und Prozesse zu kennen. Dieser Beitrag führt zunächst in Aufgabenspektrum, kommunalpolitische Institu-

\footnotetext{
L. Holtkamp ( $\varangle)$

Lehrgebiet: Politikwissenschaft IV: Politik und Verwaltung, Fern Universität Hagen, 58084 Hagen, Deutschland

E-Mail: lars.holtkamp@uni-konstanz.de

S. Grohs

Lehrstuhl für vergleichende Policy-Forschung und Verwaltungswissenschaft, Universität Konstanz, Fach D 91, 78457 Konstanz, Deutschland E-Mail: stephan.grohs@uni-konstanz.de
} 
tionen und Akteure ein, um anschließend die Ökonomisierung der Jugendarbeit und Jugendpolitik zu thematisieren.

\section{Rahmenbedingungen kommunaler Jugendpolitik}

\subsection{Aufgabenbereiche}

Kommunale Jugendpolitik umfasst Aufgaben, die von den Kommunen entweder freiwillig, als pflichtige Selbstverwaltungsaufgaben oder als übertragene Aufgaben wahrgenommen werden. Die Kinder- und Jugendhilfe als Kern der Jugendpolitik zählt dabei zu den pflichtigen Selbstverwaltungsaufgaben. Diese werden durch zahlreiche freiwillige Aktivitäten ergänzt, durch die Kommunen Jugendliche fördern, Infrastruktur bereitstellen etc. Da diese Aufgaben aus eigenen Haushaltsmitteln der Kommune erbracht werden, stehen insbesondere die freiwilligen Aufgaben in Zeiten knapper Kassen zur Disposition. Der kommunale Handlungsspielraum unterscheidet nach Aufgabenart: während in den freiwilligen Aufgaben Freiheit über das Ob und Wie der Leistungserstellung besteht, besteht bei den Selbstverwaltungspflichtaufgaben nur Handlungsspielraum über die Art und Weise der Leistungserstellung (das Wie). Im übertragenen Wirkungskreis sind die kommunalen Handlungsspielräume weitgehend eingeschränkt, da sowohl Fach- wie Rechtsaufsicht bei den staatlichen Ebenen liegen.

Der institutionelle Kern der Jugendpolitik, die Kinder- und Jugendhilfe, umfasst ein relativ breites Spektrum meist pflichtiger Selbstverwaltungsaufgaben, die von den beiden bedeutendsten Posten der Kindertagesbetreuung und den Hilfen zur Erziehung $(\mathrm{HzE})$ und verschiedenen Beratungsangeboten bis hin zur offenen Jugendarbeit sowie dem Bereich der eingreifenden Aufgaben (Wächterfunktion des Jugendamtes) reichen (vgl. Münder et al. 2006). Generelle Aufgabe der Kinder- und Jugendhilfe ist die Übernahme der kinder- und jugendbezogenen Obliegenheiten, die die Systeme der Familie und des Bildungssystems nicht zu leisten vermögen. Ziel ist die Verwirklichung des Rechtsanspruchs jedes jungen Menschen „auf Förderung seiner Entwicklung und auf Erziehung zu einer eigenverantwortlichen und gemeinschaftsfähigen Persönlichkeit" ( $\$ 1$ SGB VIII). Träger der Kinder und Jugendhilfe sind die Kreise und kreisfreien Städte als örtliche Träger ( $\$ 69$ Abs. 1 SGB VIII), allerdings können auch kreisangehörige Städte auf Antrag zu örtlichen Trägern bestimmt werden, „wenn ihre Leistungsfähigkeit zur Erfüllung der Aufgaben nach diesem Buch gewährleistet ist" ( $\$ 69$ Abs. 2 SGB VIII) (Abb. 1). 


\begin{tabular}{|c|c|c|}
\hline \multicolumn{2}{|c|}{ Eigener Wirkungskreis } & $\begin{array}{l}\text { Ubertragener } \\
\text { Wirkungskreis }\end{array}$ \\
\hline Freiwillige Aufgaben & $\begin{array}{l}\text { Selbstverwaltungs- } \\
\text { pflichtaufgaben }\end{array}$ & $\begin{array}{l}\text { Pflichtaufgaben zur } \\
\text { Erfülung nach Weisung } \\
\text { bzw. } \\
\text { Auftragsangelegenheiten }\end{array}$ \\
\hline Allg. Staatl. Rechtsaufsicht & Staatl. Rechtsaufsicht & $\begin{array}{l}\text { Staatl. Rechts- und } \\
\text { Fachaufsicht }\end{array}$ \\
\hline $\begin{array}{l}\text { Finanzierung aus Haushaltsmitteln } \\
\text { der Kommune }\end{array}$ & $\begin{array}{l}\text { Primäre Finanzierung aus } \\
\text { Haushaltsmitteln der } \\
\text { Kommune }\end{array}$ & $\begin{array}{l}\text { Finanzierung aus Bundes- } \\
\text { Landesmitteln }\end{array}$ \\
\hline $\begin{array}{l}\text { Beispiele: } \\
\text {-Jugendtreffs } \\
\text {-Jugendfreizeiten } \\
\text { Förderung von Vereinen, } \\
\text { Jugendgruppen etc. }\end{array}$ & $\begin{array}{l}\text { - Hilfen zur Erziehung } \\
\text { - Kindertagesbetreuung } \\
\text { - Sozialhilfe } \\
\text { - Allgemeiner Sozialer Dienst }\end{array}$ & $\begin{array}{l}\text { - Bewilligung und } \\
\text { Wohngeld } \\
\text { - Gesundheitsaufsicht, } \\
\text { Seuchenbekämpfung }\end{array}$ \\
\hline (Ob und wie) & $\begin{array}{l}\text { Lokale Autonomie } \\
\quad(\text { Wie })\end{array}$ & (Weder ob noch wie) \\
\hline
\end{tabular}

Abb. 1 Überblick über diese Aufgabenfelder kommunaler Jugendpolitik

Den rechtlichen Rahmen bildet das Kinder- und Jugendhilfegesetz (KJHG) von 1990 (eingegliedert in das SGB als SGB VIII). Mit dem KJHG, das das Jugendwohlfahrtsgesetz (JWG) ersetzt, trat an die Stelle obrigkeitlicher Fürsorge durch das Jugendamt eine Orientierung am Bild des Jugendamtes als Dienstleistungsträger, dem gegenüber in großem Umfang Rechtsansprüche geltend gemacht werden können (vgl. Münder et al. 2006). Das neue Gesetz brachte als wesentliche Veränderungen eine Konzentration der Zuständigkeiten der Jugendhilfe bei den örtlichen Jugendämtern, einen Abbau von Maßnahmen mit eingreifendem Charakter und einer umfassenden Benennung allgemeiner Fördermaßnahmen und präventiver Leistungen sowie Elemente der Partizipation und Wahlfreiheit der Klienten. Als wesentliche Aufgaben der Kinder- und Jugendhilfe nennt das KJHG einerseits die Leistungen der Jugendhilfe ( $\$ 11-41$ SGB VIII), die weitgehend als pflichtige Selbstverwaltungsaufgaben $\mathrm{zu}$ fassen sind, und die so genannten „anderen Aufgaben“ ( $\$ 42-60)$, die keine inhaltliche Systematik aufweisen (vgl. Münder et al. 2006), jedoch durchwegs den Charakter uneingeschränkter Pflichtaufgaben haben, von denen die gesetzliche Inobhutnahme und die Herausnahme des Kindes oder Jugendlichen aus der Familie ohne Zustimmung des Erziehungsberechtigten Aufgaben der Ordnungsverwaltung beinhalten. 
Wie auch immer die Reform des Jugendhilferechts im Detail einzuschätzen ist, besteht Konsens darüber, dass die Kommunen durch die Einführung des KJHG 1990 in erheblichem Maße Handlungsspielräume einbüßten. Neben vielen normierten Pflichtaufgaben wurden individuell einklagbare Rechtsansprüche im Gesetz verankert, die erhebliche finanzielle Belastungen für die Kommunen mit sich brachten und dazu führten, dass viele individuell nicht einklagbare Aufgaben angesichts des eher schrumpfenden Gesamtbudgets im Zuge der Haushaltskrise nur noch begrenzt wahrgenommen werden konnten. Hinzu kamen in den vergangenen Jahren insbesondere im Bereich der Kindertagesbetreuung eine Ausdehnung der Pflichtleistungen sowohl hinsichtlich des Kreises der zu betreuende Kinder (Unter Dreijährige) als auch im zeitlichen Umfang der Betreuung (Ganztagsbetreuung) im Tagesbetreuungsausbaugesetz (TAG) von 2005 und Kinderförderungsgesetz (KiföG) von 2008. Generelles Politikum im Zuge der Schaffung dieser neuen Leistungen ist die Finanzierung der Aufgaben und der mit ihr verbundenen Verwaltungs- und Infrastrukturaufwandes. Generell gilt als Maßstab das Konnexitätsprinzip nach dem Motto „Wer bestellt zahlt!“. Auch nach Einführung des Konnexitätsprinzips in das Grundgesetz (Art. 104a GG) bleibt die Umsetzung defizitär, wie sich an der Umsetzung des Kinderförderungsgesetzes (KiföG) von 2008 zeigt. Zwar stellt der Bund 4 Mrd. € zur Verfügung, einzelne Bundesländern verweigern jedoch bislang ihren zugesicherten Beitrag anzuerkennen (vgl. Deutscher Städtetag 2010, S. 17). Als zusätzliche Erschwernis einer konsequenten Konnexitätspolitik erweisen sich die Regelungen der Föderalismusreform, nach der durch die Neuregelung des Art. 84 Abs. 1 Satz 7 Grundgesetz dem Bund künftig untersagt ist, über die Trägerschaft und Finanzierung von Einrichtungen zu entscheiden und hier auf die Länder angewiesen ist.

\subsection{Finanzierung}

Die Finanzierung von Jugendpolitik erfolgt zum Großteil aus den kommunalen Haushalten. Zu einem kleineren Teil werden - in der Regel projektbezogen - Mittel der Länder oder des Bundes (z. B. für die Kooperation Schule-Jugendhilfe) oder der EU ( $z$. B. Jugendberufshilfe) bereitgestellt. Die Länder sind zusätzlich im Rahmen ihrer Unterstützungsfunktion für den gleichmäßigen Ausbau örtlicher Einrichtungen und Angebote ( $\$ 82$ SGB VIII) verpflichtet, zusätzlich fördernd tätig zu werden.

Die Bedeutung der Jugendpolitik im kommunalen Gesamtgefüge lässt sich an den durch sie gebundenen Ressourcen in den Kommunen ablesen. Gemäß dem Gemeindefinanzbericht für 2011 (vgl. Anton und Diemert 2011) wurden 2010 


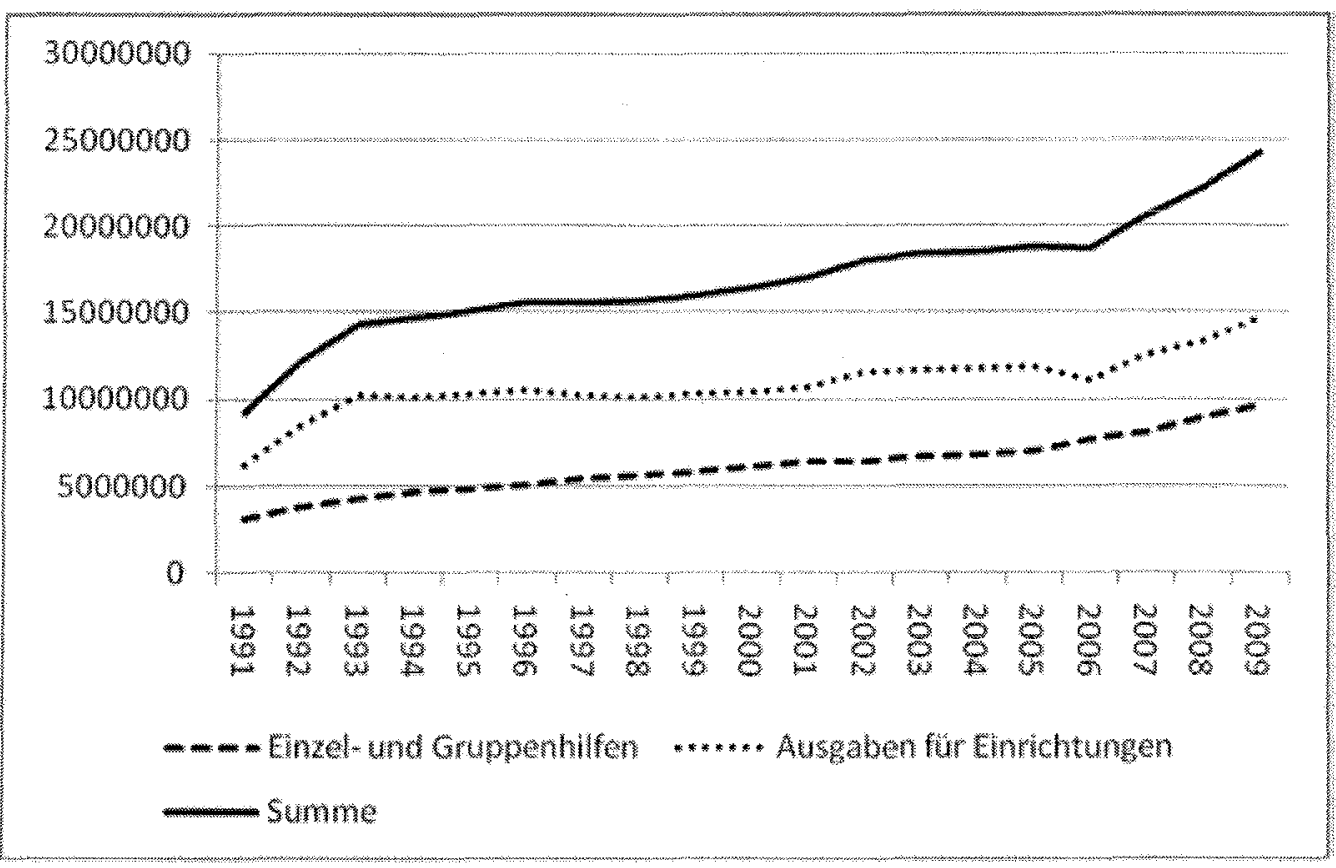

Abb. 2 Gesamtausgaben der Jugendhilfe (in $1.000 €$ ). (Quelle: Statistisches Bundesamt 2011: Statistiken der Kinder- und Jugendhilfestatistiken. Ausgaben und Einnahmen. Wiesbaden)

6,5 Mrd. $€$ für Jugendhilfeaufgaben ausgegeben, das sind immerhin 4,8 \% der gesamten kommunalen Ausgaben. Dabei sind die Aufwendungen für kommunales Personal und Sachmittel im Bereich Verwaltung und der sozialen Dienste noch nicht enthalten. Im Gegensatz zu anderen Haushaltsposten sind die Jugendhilfeausgaben die vergangenen Jahre konstant gestiegen (vgl. Abb. 2). Hier lassen sich insbesondere die Auswirkungen der Ausbau der Kindertagesbetreuung ablesen, die seit 2006 haushaltswirksam werden.

Die kommunalen Finanzen stützen sich auf der Einnahmeseite einerseits auf Steuereinnahmen, dies sind insbesondere die Gewerbesteuer und der Anteil an der Einkommensteuer, die 2009 mit zusammen $49 \mathrm{Mrd}$. $€$ rund $30 \%$ der kommunalen Einnahmen ausmachten. Hinzu kommen insbesondere Zuweisungen von Ländern und Bund sowie Gebühreneinnahmen. Die Steuereinnahmen erwiesen sich in der Vergangenheit als äußerst konjunkturabhängig. So ging das Gewerbesteueraufkommen im Krisenjahr 2009 um 19,7\%, der Einkommensteueranteil um 7,7 \% zurück, im Jahr 2010 nochmals um weitere 5,6 bzw. 7,0 \% (Anton und Diemert 2010, S. 11). Diesem Rückgang auf der Einnahmeseite stand eine deutliche Zunahme der sozialen Leistungen gegenüber, deren Volumen 2009 erstmals die $40 \mathrm{Mil}$ liardengrenze überschritt und die um 4,9\% im Jahr 2009 und 4,4\% im Jahr 2010 


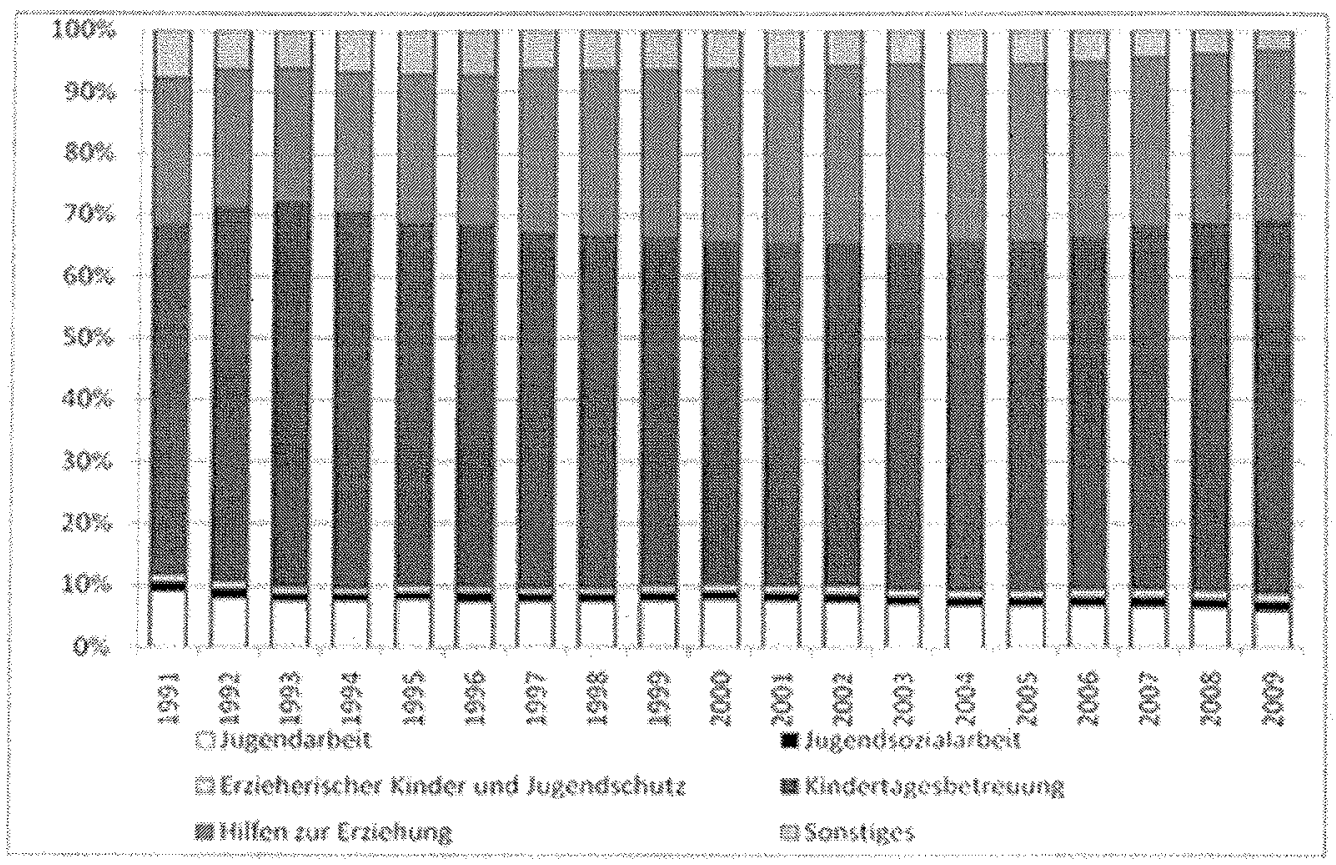

Abb. 3 Ausgaben für Kinder- und Jugendhilfe anteilig nach Teilgebieten. (Quelle: Statistisches Bundesamt 2011: Statistiken der Kinder- und Jugendhilfestatistiken. Ausgaben und Einnahmen. Wiesbaden)

gestiegen sind (Anton und Diemert 2010, S. 11). Diese Steigerungen sind einerseits auf die Zunahme der Fälle der Kosten für Unterkunft nach SGB II zurückzuführen; daneben spielen aber auch Steigerungen im Bereich der Eingliederungshilfe und der Grundsicherung im Alter. Besonders deutlich war der Anstieg in der Kinder und Jugendhilfe mit 10,4\%. Dabei ist zu beachten dass Eingliederungshilfe, Grundsicherung im Alter und Jugendhilfe konjunkturunabhängig ansteigen und dies aller Voraussicht nach auch weiter tun werden.

So öffnet sich in zahlreichen Kommunen zunehmend die Schere zwischen Einnahme und Ausgaben (vgl. Holtkamp 2010 für weitere Details). Für die Jugendpolitik bedeutet dies eine zunehmende Verschiebung der eingesetzten Mittel von freiwilligen Leistungen wie der offenen Jugendarbeit und der Förderung von Vereinen und Jugendverbänden hin zu den teuren Pflichtleistungen im Bereich der Hilfen zur Erziehung und Kindertagesbetreuung, wie sich deutlich in Abb. 3 ablesen lässt. Der Anteil für Jugendarbeit, Jugendsozialarbeit und sich im Posten „Sonstiges“ versteckenden freiwillige Leistungen hat sich von rd. $20 \%$ der Ausgaben im Jahr 1991 hat sich bis zum Jahr 2010 halbiert.

Kommunale Jugendpolitik ist dadurch vor allem durch das Auseinanderklaffen zwischen Bedarf und zur Verfügung stehenden Mitteln betroffen. Gerade Städte mit 
hoher Belastung durch Arbeitslosigkeit und andere Problemlagen weisen gleichzeitig ein Einnahmedefizit - insbesondere durch geringere Gewerbesteuereinnahmen- auf, das gerade in Zeiten der Finanzkrise weiter anwächst. Je höher also der Bedarf ist, desto geringer die vorhandenen kommunalen Mittel - dies gilt sowohl im interkommunalen Vergleich als auch in zeitlicher Perspektive. Bei schrumpfendem finanziellem Handlungsspielraum werden folglich die internen Verteilungskämpfe stärker. Wie diese Verteilungskämpfe ausgehen, hängt nicht unwesentlichen von den kommunalpolitischen Akteuren und den kommunalpolitischen Entscheidungsstrukturen $\mathrm{ab}$, in die in den folgenden Abschnitten eingeführt wird.

\subsection{Akteure}

Als jugendpolitischen Akteure treten auf kommunaler Ebene zunächst die eigentlichen „politischen“ Akteure, die kommunalen Ratspolitiker und die mittlerweile in allen Bundesländern direkt gewählten Bürgermeister auf. Neben diesen haben Angehörige der Kommunalverwaltung, freie Träger und Vereine, aber auch die Betroffenen, die Kinder- und Jugendlichen selbst Einfluss auf die Politikgestaltung, etwa die vielerorts aktiven Jugendgemeinderäte. Der Einfluss unterscheidet sich dabei allerdings erheblich. Die Entscheidungs-, der Implementations- und Durchführungsstrukturen sind eng verwoben. Die Akteurskonstellationen in der Kinderund Jugendhilfe zeichnen sich durch eine Dominanz freier Träger in der Leistungserbringung, also der Trägerschaft von Jugendtreffs, Kindergärten und Jugendheimen aus. Als freie Träger werden Organisationen bezeichnet, die nicht staatlich oder kommunal sind, aber einen Teil ihrer Aufgaben übernehmen, in der Regel gehören diese zu den sechs Spitzenverbänden der freien Wohlfahrtspflege. Die Wohlfahrtsverbände bzw. die ihnen angeschlossenen Einrichtungen und Dienste sind nach wie vor die wichtigsten Leistungserbringer in der lokalen Jugendpolitik. Von den anderen lokal aktiven Vereinen, Selbsthilfegruppen oder kommerziellen Anbietern unterscheiden sie sich durch ihre Organisation auf nationaler und regionaler Ebene. Neben der Bedeutung als Leistungserbringer zeichnen sie sich durch ihren expliziten Interessenvertretungsanspruch und den hohen Grad an Einbindung in staatliche Politiken und schließlich ihre Gebundenheit an die verbandsspezifischen Werte und Weltanschauungen aus. Die sechs in der Bundesarbeitsgemeinschaft der freien Wohlfahrtspflege (BAGFW) organisierten Spitzenverbände der freien Wohlfahrtspflege - Arbeiterwohlfahrt (AWO), Deutscher Caritas Verband (DCV), Diakonisches Werk (DW), Deutscher Paritätischer Wohlfahrtsverband, Deutsches Rotes Kreuz (DRK) und die Zentralwohlfahrtsstelle der Juden 
(ZWSt) - unterscheiden sich dabei sowohl hinsichtlich ihres Leistungsportfolios, der internen Organisation wie ihrer weltanschaulichen Bindung.

Aktuell arbeiten in über 100.000 Einrichtungen und ambulanten Angeboten der Wohlfahrtsverbände über 1,5 Mio. Beschäftigte, in denen fast $4 \mathrm{Mio}$. Menschen gepflegt, versorgt, betreut, aktiviert und beraten werden (Zahlen für 2008; BAGFW 2009). Die Wohlfahrtsverbände sind damit seit den 70er Jahren zu einem der größten Arbeitgeber Deutschlands avanciert, der quantitativ betrachtet nicht den Vergleich mit großen internationalen Konzernen scheuen muss.

Die Wohlfahrtsverbände erfüllen v. a. drei Funktionen in der kommunalen Jugendpolitik: Sie sind professionelle Dienstleistungsanbieter (Dienstleistungsfunktion), sie organisieren eine große Zahl von ehrenamtlichen Helfern (Engagementgenerierungsfunktion) und schließlich vertreten sie die Interessen ihrer Klientel und fungieren insofern als ein sozialpolitischer Anwalt (Anwaltsfunktion bzw. advokatorische Interessenvertretung). In der Praxis ist aber davon auszugehen, dass v. a. die erste Funktion von entscheidender Bedeutung für das Verhältnis von öffentlichen und freien Trägern ist. Zwar wird zunehmend die Relevanz von ehrenamtlichem Engagement für die kommunale Sozialpolitik gesehen, deutlich wird für Kommunalpolitik und -verwaltung aber auch, dass die Wohlfahrtsverbände gerade im Zuge des Wertewandels sowie einer zunehmenden Professionalisierung und Dienstleistungsorientierung immer mehr Probleme bei der Rekrutierung von Ehrenamtlichen haben. Auch die Anwaltsfunktion der Wohlfahrtsverbände ist in den Jugendhilfeausschüssen vor Ort nur selten zu beobachten, es sei denn, sie geht mit der Forderung nach zusätzlicher Förderung einher.

Über die besonderen Entscheidungskonstellationen in den Jugendhilfeausschüssen (s. u.) sind die freien Träger an den Entscheidungen über die Ausgestaltung der Jugendhilfe beteiligt. Gleichzeitig werden die Kommunalverwaltungen nicht nur als Gewährleister, sondern auch als eigenständige Leistungserbringer neben freien Trägern, Vereinen, und privatgewerblichen Anbietern tätig. Trotz dieser grundsätzlichen Verwobenheit ist es sinnvoll, die Akteure auf der „Input-Seite“, also den kommunalen Entscheidungsstrukturen, von den letztendlichen Leistungserbringern zu trennen. Dominante Akteure auf der Entscheidungsseite bleiben die Kommunalverwaltungen und die kommunalen Vertretungskörperschaften. Hier ist insbesondere auf das Verhältnis zwischen Verwaltungsspitze und den Fachverwaltungen zu achten. Auf der Seite der Leistungserbringer sind insbesondere die Träger der freien Wohlfahrtspflege, aber auch sonstige Initiativen, Vereine und andere Akteure des „Dritten Sektors“ sowie privatwirtschaftliche Akteure von Bedeutung. Neben den Wohlfahrtsverbänden sind auf kommunaler Ebene noch die Jugendverbände von gewisser Bedeutung. Sie sind in der Regel auf das Teilgebiet der verbandlichen und offenen Jugendarbeit spezialisiert und sind als stimmbe- 
rechtigte Mitglieder in zahlreichen Jugendhilfeausschüssen vertreten, häufig unter dem Dach der sogenannten „Jugendringe“ (vgl. Münchmeier 1995). Sie zeichnen sich gegenüber den Wohlfahrtsverbänden durch ihre altersmäßig klar eingegrenzte Zielgruppe, den Charakter der Selbstorganisation und eine Orientierung an Freizeitgestaltung statt Problembearbeitung aus (vgl. Merchel 2003, S. 146 f.).

\subsection{Entscheidungsstrukturen: Der Jugendhilfeausschuss}

Zentrales jugendpolitisches Entscheidungsgremium ist der Jugendhilfeausschuss (JHA), der in mehrfacher Hinsicht eine Besonderheit unter den kommunalpolitischen Gremien darstellt. Nach $\$ 70$ SGB VIII werden die Aufgaben des Jugendamtes durch den Jugendhilfeausschuss und durch die Verwaltung des Jugendamtes in gemeinsamer Verantwortung wahrgenommen (Prinzip der Zweigliedrigkeit ( legialverfassung“)): Die Jugendamtsverwaltung führt die laufenden Geschäfte, der Jugendhilfeausschuss befasst sich „mit allen Angelegenheiten der Jugendhilfe" ( $\$ 71$ Abs. 2 SGB VIII)). Dies stellt eine Besonderheit in der deutschen verwaltungsrechtlichen Tradition dar, da das Jugendamt die einzige kommunale Behörde ist, deren Organisation durch Bundesrecht vorgegeben ist und somit der Organisationshoheit der Kommunen entzogen ist. Zudem ist der Jugendhilfeausschuss unter demokratietheoretischem Aspekt ein besonderes Element, da in ihm neben den gewählten Vertretern der Gebietskörperschaft zwei Fünftel der stimmberechtigten Mitglieder aus dem Bereich der anerkannten freien Träger und der Jugendverbände kommen (vgl. Münder und Ottenberg 1999).

Neben den mindestens 10 stimmberechtigten Mitgliedern sind (unterschiedlich nach den Ausführungsbestimmungen der Bundesländer) auch nicht stimmberechtigte Mitglieder zu berufen. In NRW sind dies beispielsweise ein Hauptverwaltungsbeamter, der Leiter des Jugendamtes, ein Mitglied eines Jugend-, Familienoder Vormundschaftsgerichts, ein Vertreter der Arbeitsverwaltung, ein Vertreter der Schulen, ein Vertreter der evangelischen und katholischen Kirche und ein Vertreter der Polizei (zu der Zusammensetzung in anderen Bundesländern, Münder und Ottenberg 1999, S. 41 ff.). Weitere nicht stimmberechtigte Mitglieder können die Kommunen noch über die Jugendamtssatzung bestimmen. In der kommunalen Praxis hat der Jugendhilfeausschuss damit häufig mehr Mitglieder als die anderen Fachausschüsse. Durch diese besonderen Regelungen kommt es zur Situation, dass es im JHA möglich ist, Mehrheiten zu finden, die sich von der Mehrheitssituation im Rat unterscheiden. Insofern kommt den Verbänden bei knappen politischen Mehrheiten de facto eine Veto-Position zu (vgl. Bogumil und Holtkamp 2006, S. 167). Zudem entsteht so die Situation. dass „die freien Verbände [...] unmittel- 
Tab. 1 Anteile einzelner Trägergruppen in Kinder- und Jugendhilfeausschüssen. (Quelle: Pluto et al. 2007, S. 323 (Tab. 8.1) leicht verändert)

\begin{tabular}{lllll} 
& 2000 & \multicolumn{3}{c}{2004} \\
\cline { 2 - 5 } & Ost (\%) & West (\%) & Ost (\%) & West (\%) \\
\hline Wohlfahrtsverbände & 61 & 52 & 53 & 50 \\
\hline Jugendringe und Jugendverbände & 22 & 40 & 29 & 39 \\
Andere freie Träger & 16 & 7 & 13 & 10 \\
\hline Kirchen & 1 & 1 & 2 & 2 \\
\hline
\end{tabular}

bar über die Verausgabung und Verteilung öffentlicher Haushaltsmittel (und somit über einen Teil ihrer Einnahmen) mit [entscheiden]" (Ronge 1993, S. 339; vgl. auch Merchel und Reismann 2004, S. 205; Bogumil und Holtkamp 2006, S. 167).

In der Realität der Jugendhilfeausschüsse dominieren die etablierten Spitzenverbände der freien Wohlfahrtsverbände (vgl. Tab. 1). Dies führt in der Regel zum Ausschluss kleiner Initiativen und Vereine. Münder und Ottenberg (1999, S. 78) nennen dies eine „Geschlossene Gesellschaft". Zudem kommen zu den offiziellen Verbandsvertretern versteckte Verbandsvertreter, d. h. kommunalpolitisch aktive Vertreter von Wohlfahrtsverbänden, die qua Ratsmandat in den Ausschüssen sitzen (vgl. Seckinger et al. 1998, S. 163).

Die Vetoposition der Verbände im JHA kann dazu führen, dass der Parteienwettbewerb, der in vielen anderen kommunalen Gremien stark ausgeprägt ist, hier kaum eine Rolle spielt. Der Einfluss des JHA wird allerdings durch die Dominanz der Verwaltung, gerade in kostenträchtigen Feldern relativiert. Es ist häufige Praxis, dass bei den „teuren“ Entscheidungen die Verwaltungsseite die Entscheidungen dominiert (vgl. Münder und Ottenberg 1999) und dem JHA nur eine nachgeordnete Rolle zukommt. Zudem finde wesentlichen Entscheidungen in sog. Vorentscheiderkreisen (Banner 1982) statt. Verhandlungen laufen eher im Vorfeld der öffentlichen Sitzungen des JHA ( $z$. B. auf den Fraktionssitzungen, an denen häufig auch die von der jeweiligen Fraktion nominierten freien Träger teilnehmen) oder in den sog. Arbeitsgemeinschaften im Rahmen der Jugendhilfeplanung als eher fachlich orientierte Gremien ab.

Im JHA zeigt sich dagegen wie in vielen anderen Fachausschüssen, dass das einst von dem Satiriker Parkinson entwickelte Gesetz der Ausschusstätigkeit auch heute noch teilweise aktuell ist: „Die auf einen Tagesordnungspunkt verwendete Zeit ist umgekehrt proportional zur Größe der Summe, um die es geht" (Parkinson 1994, S. 49).

Hinzu kommt, dass die Lokalpresse komplexere 'Themen wie die Erziehungshilfe kaum aufnimmt, sondern sich vor allem auf die Angebote der offenen Jugend- 
arbeit und Kinderbetreuung konzentriert. Auffällig ist, dass im Gegensatz zu den bundesweiten Medien in der Lokalpresse kaum über die typischen Problemthemen berichtet wird (Drogen, Kriminalität, Jugendarbeitslosigkeit, sexueller Missbrauch etc.) und dass diese Themen auch in den Jugendhilfeausschüssen eher selten behandelt werden (Straub 2004, S. 36; Merchel und Reismann 2004, S. 169 f.). Gerade für die Lokalpresse ist hier wie in den meisten Politikfeldern eine wenig problembezogene und konfliktscheue Berichterstattung typisch.

\section{3 Ökonomisierung der Jugendarbeit und Jugendpolitik?}

In den vergangenen Jahren haben Kommunalpolitik und -verwaltung eine Reihe von Veränderungsprozessen mitgemacht, die Entscheidungsprozesse und Leistungsprozesse einer deutlichen Veränderung unterzogen haben. Hierzu gehören insbesondere managementorientierte Reformen, die unter der Bezeichnung „Neues Steuerungsmodell" (NSM) in zahlreichen Kommunen eingeführt wurden, um Haushaltsprobleme und wahrgenommene Steuerungsdefizite zu beheben. In diesem Zusammenhang wurde auch die herkömmlichen Leistungsbeziehungen zwischen öffentlichen und freien Trägern auf eine neue, häufig als „Ökonomisierung“ bezeichnete, Grundlage gestellt (vgl. Grohs 2010).

Unter dem zunehmenden Problemdruck der kommunalen Haushalte und dem Eindruck internationaler Vorbildkommunen hat die Kommunale Gemeinschaftsstelle für Verwaltungsvereinfachung (KGSt) seit Anfang der 1990er Jahre in einer Reihe von Berichten ihre Konzeption des „Neuen Steuerungsmodells" entwickelt. Dabei sollte die Effizienz und Effektivität der Kommunalverwaltung durch eine umfassende Dezentralisierung von Fach- und Ressourcenverantwortung und eine output-gesteuerte Verwaltungsführung erreicht werden. Diese sollte durch eine Ungestaltung der Aufbauorganisation (Fachbereichsstrukturen), die Einführung betriebswirtschaftlicher Steuerungsinstrumente (Budgetierung, Controlling, Kosten-Leistungsrechnung) mit „Produkten“ als zentralen Informationsträgern und einem prononcierten Personalmanagement unterstützt werden. Sowohl interne Verwaltungsabläufe, städtische Beteiligungen als auch die Steuerung der Verwaltung durch die Gemeindevertretungen sollten mittels Kontrakten und Zielvereinbarungen erfolgen. Dabei soll das Verhältnis zwischen Rat bzw. Jugendhilfeausschuss und Verwaltung von der kurzfristigen Logik der Detaileingriffe auf eine mittelfristige strategische Steuerung umgestellt werden: Der Rat solle über das "Was“, die Verwaltung eigenverantwortlich über das „Wie“ des Verwaltungshandelns bestimmen. „Unter Strom gesetzt“ (KGSt 1995, S. 22) werden sollte dieses „Neue“ 
System der Verwaltungssteuerung durch den Einsatz von Wettbewerbsmechanismen (sowohl verwaltungsintern als auch nach außen) sowie eine Orientierung am „Kunden“, ergo dem Bürger in der Rolle als „Nachfrager“ öffentlicher Dienstleistungen (vgl. Jann 2005).

Die KGSt propagierte das NSM als Lösung für alle lokalen Politikfelder und Aufgabenbereiche: „Das Neue Steuerungsmodell sieht in der Steuerung des Fachbereichs Jugend und Soziales keinen Unterschied zur Steuerung der kommunalen Verkehrsgesellschaft" (KGSt 1995, S. 28-29). Obwohl gerade das Jugendamt als exemplarisches Beispiel für Instrumente der Neuen Steuerung diente, blieben die Besonderheiten der Jugendhilfe dabei zunächst außen vor. Diese „universalistische“ Sichtweise wurde zu Recht kritisiert und auf die Spezifika der Kinder- und Jugendhilfe (hingewiesen (vgl. Merchel 1996, S. 213-214).

Für die öffentlichen Träger am Beispiel der Jugendämter liegt eine ausführliche Evaluationsstudie auf der Basis einer repräsentativen Befragung und vertiefter Fallstudien nach über zehn Jahren Praxiserfahrungen vor (vgl. Bogumil et al. 2007; Grohs 2007, 2010). Diese zeigt ein zwiespältiges Bild von Umsetzung und Wirkungen des NSM: Es gab in den deutschen Kommunen eine breite Verwaltungsmodernisierungsbewegung. Der KGSt ist es gelungen, die intensive Beschäftigung mit einer im Kern betriebswirtschaftlich ausgerichteten Binnenmodernisierung in den deutschen Kommunen zu initiieren, obwohl sie lediglich beratende Funktionen hat und so den Kommunen das NSM nicht rechtlich vorschreiben konnte.

Über 87 \% der antwortenden Jugendämter geben an, seit den 1990er-Jahren Maßnahmen im Rahmen der Verwaltungsmodernisierung durchgeführt zu haben. Dabei haben sich insgesamt über $73 \%$ der befragten Jugendämter ganz (17\%) oder teilweise (56 \%) am NSM der KGSt orientiert. Der tatsächliche Modernisierungsstand und die Modernisierungsergebnisse in den deutschen Kommunen sind allerdings sehr unterschiedlich. Es zeigt sich, dass auf der Ebene der einzelnen Modernisierungsinstrumente kein einziges von mehr als der Hälfte der Kommunen umgesetzt wurde. Dabei liegt der Schwerpunkt der Modernisierungsmaßnahmen in der Einführung des Produktkonzeptes, der Dezentralisierung der Fach- und Ressourcenverantwortung sowie der Budgetierung in jeweils rund $40 \%$ der Jugendämter. Auffällig ist, dass häufig Verantwortungsstrukturen dezentralisiert wurden, der Aufbau entsprechender Controllingverfahren aber stockt. Diese angesichts der anfangs angeführten hohen Modernisierungsabsichten und der Vehemenz der fachlichen Diskussionen ernüchternden Zahlen deuten darauf hin, dass das NSM insgesamt mehr als Werkzeugkasten denn als holistisches Reformkonzept betrachtet wurde.

Auch hinsichtlich der erhofften Wirkungen bleibt die Bilanz ambivalent: Bezogen auf das Ziel der Wirtschaftlichkeitssteigerung und Kostenreduzierung ist die Re- 
formbilanz eher ernüchternd. Es gibt kaum stichhaltige Anhaltspunkte dafür, dass das NSM nachhaltig zur Haushaltskonsolidierung beigetragen hat, dass Einsparungen größerer Dimension und längerfristiger Wirksamkeit erzielt werden konnten oder dass der Ressourcenaufwand in der Gesamtverwaltung vermindert werden konnte, insbesondere dann, wenn man die nicht unerheblichen Transaktionskosten der Reform in Rechnung stellt. Die NSM-Reform hat einige Verbesserungen im Bereich der Bürger- und Klientenorientierung (Servicequalität, ganzheitliche Fallbetreuung) bei den öffentlichen Trägern begünstigt und einer verstärkten Thematisierung von Qualitätsaspekten der sozialen Dienste (z. B. durch Einführung eines Qualitätsmanagements). Zwar wären diese prinzipiell auch ohne NSM erreichbar gewesen, aber die Verwaltungsmodernisierung hat ein günstiges Reformklima geschaffen, so dass aus früheren Diskurskontexten stammende Reformkonzepte wie regionalisierte Anlaufstellen oder integrierte Hilfen jetzt nachhaltig implementiert wurden. Die vielfältigen Bemühungen um eine verbesserte Outputsteuerung haben die Transparenz des Verwaltungshandelns ohne Zweifel erhöht, ohne dass es zu einer wirklichen Ablösung der „klassischen“ Input- und Regelsteuerung gekommen ist. Viele öffentliche Träger haben bessere Kenntnisse im Hinblick auf Verwaltungsleistungen, Kosten und wichtige „Wirtschaftskennzahlen“ der Verwaltung. Dies hat jedoch nicht zu relevanten Veränderungen in der politischen und administrativen Steuerung der Kommunen geführt. Eine klare Trennung von Rollen und Verantwortlichkeiten findet selten statt.

Auch die interorganisatorische Entwicklung der Ökonomisierung insbesondere im Verhältnis zwischen Kommune und den freien Trägern ist in der Jugendpolitik nicht sehr ausgeprägt. Kontraktmanagement und Leistungsvereinbarungen wurden mit den Zielen eingeführt, die Trägerstrukturen vermehrt über die Leistungsseite zu steuern, dabei von den freien Trägern vermehrt Rechenschaft über Qualität und Kosten einzufordern und sie damit vermehrt unter Wettbewerb zu setzen. Mit letzterem war auch der Anspruch einer Pluralisierung der Trägerlandschaft verbunden. Die formelle Umsetzung von Kontraktmanagement ist nach allen vorliegenden Informationen auch relativ weit fortgeschritten (vgl. Dahme et al. 2005). Eine Umfrage unter den deutschen Jugendämtern ergab, dass in rund $70 \%$ der Jugendämter inzwischen mit Leistungsvereinbarungen gearbeitet wird und rund ein Drittel im Rahmen der NSM-Modernisierung ein Kontraktmanagement mit freien Trägern etabliert hat (Grohs 2010). Hinsichtlich der Ausgestaltung ist allerdings davon auszugehen, dass in einer Großzahl der Fälle die Einführung nur formal ist und wesentliche Modernisierungsimpulse ausbleiben. So deutet wenig auf eine tiefgreifende Pluralisierung der Anbieterstruktur hin - im Gegenteil scheint Kontraktmanagement häufig zu einer Verfestigung der Anbieterstrukturen zu führen (vgl. Heinze et al. 1997; Dahme et al. 2005, S. 54; Grohs 2010). Ebenso bleiben 
Wettbewerbsimpulse die Ausnahme, in weniger als der Hälfte der Fälle der Jugendamtsbefragung werden beispielsweise im Rahmen der Vergabe von Leistungsvereinbarungen Qualitäts- und Kostenvergleiche angestellt. In weiten Teilen scheinen also die Leistungsvereinbarungen allenfalls eine Scheinökonomisierung darzustellen, während sich die etablierten freien Träger „einen großen Teil des Kuchens“ weiterhin über ihre traditionell engen Beziehungen zur Verwaltung und Kommunalpolitik und ihre umfassenden Beteiligungsrechte im Jugendhilfeausschuss und in der Jugendhilfeplanung sichern.

Kosteneinsparungen werden so nicht durch Wettbewerbsverfahren, sondern durch hierarchische Budgetdeckelungen ohne Rückkoppelung an sozialpolitische Zielsetzungen durch Bürgermeister und Kämmerer durchgesetzt (vgl. Dahme et al. 2005, S. 112). Auch die Implementation von Qualitätssicherungselementen im Kontraktmanagement ist weiterhin defizitär: Hierauf deuten etwa Merchels Untersuchung von Qualitätsentwicklungsvereinbarungen nach $\$ \$ 98$ a-g SGB VIII hin, in denen er zeigt, dass der überwiegende Teil der analysierten Vereinbarungen nicht oder unzureichend ausformuliert sind und somit kaum zu einer tatsächlichen Qualitätssteuerung beitragen können. Die Vereinbarungen scheinen daher eine reine Legitimationsfunktion zu besitzen (Merchel 2006).

\section{Fazit: Jugendarbeit unter Konsolidierungsdruck}

Die Hoffnung, dass sich durch effizientere Steuerungsmodelle Leistungen und Qualität in der Jugendarbeit bei gleichzeitigen Einsparungen steigern lassen, hat sich häufig in der Implementation vor Ort als trügerisch erwiesen. Nicht selten kam es eher zur einer Scheinökonomisierung und einer lediglich symbolischen Umsetzung von Qualitätssicherungsmaßnahmen, die zwar aufwändige Berichte, Vereinbarungen und Indikatorensysteme zur Folge hatten, die aber kaum Einfluss auf die empirisch beobachtbaren Entscheidungsprozesse hatten. Hinter der Fassade der neuen Managementsysteme wirken häufig noch dieselben Kräfte wie vor der Einführung. Einsparungen werden meist in der offenen Jugendarbeit, die nicht durch einklagbare Rechte der Klienten abgesichert ist, nach dem Rasenmäherprinzip durch Druck der Verwaltungsspitze vorgenommen, während der weit größere Teil des Haushalts im Bereich der Erziehungshilfe und Kindertagestätten nicht zur Disposition steht. Die Ausgaben in diesen Bereichen dürften durch diversen Leistungsgesetze und sozialstrukturelle Entwicklungen vielmehr weiter steigen und werden damit indirekt den Konsolidierungsdruck auf die offene Jugendarbeit weiter erhöhen. Dies entspricht zum Teil notgedrungen durchaus auch den Präfe- 
renzen der freien Träger, die sich unter dem Druck rückläufiger Kirchensteuereinnahmen von diesem „defizitären Geschäftsbereich“ bereitwillig trennen, um sich stärker beispielsweise auf die kostendeckende Erziehungshilfe zu konzentrieren. Damit stehen die Strukturen der offenen Jugendarbeit weiterhin unter einem extremen Kostendruck, insbesondere in größeren Kommunen mit ausgeprägten sozialstrukturellen Problemen, in denen sich die kommunale Haushaltskrise voraussichtlich noch weiter verschärfen wird.

\section{Literatur}

Anton, S., \& Diemert, D. (2010). Kommunale Finanzen: Kein Licht am Ende des Tunnels! Der Gemeindefinanzbericht 2010 im Detail. Der Städtetag, 11-85.

Anton, S., \& Diemert, D. (2011). Weniger Defizite - aber die Strukturkrise bleibt. Gemeindefinanzbericht im Detail. Der Städtetag, 11-88.

Banner, G. (1982). Zur politisch-administrativen Steuerung in der Kommune. Archiv für Kommunalwissenschaften, 21, 26-47.

Bogumil, J., \& Holtkamp, L. (2006). Kommunalpolitik und Kommunalverwaltung. Eine policyorientierte Einführung. Wiesbaden: VS Verlag für Sozialwissenschaften.

Bogumil, J., Grohs, S., Ohm, A. K., \& Kuhlmann, S. (2007). Zehn Jahre Neues Steuerungsmodell. Eine Bilanz kommunaler Verwaltungsmodernisierung. Berlin: Edition Sigma.

Bundesarbeitsgemeinschaft der freien Wohlfahrtspflege (BAGFW). (2009). Gesamtstatistik 2008. Berlin: BAGFW.

Dahme, H.-J., Kühnlein, G., Wohlfahrt, N., \& Burmester, M. (2005). Zwischen Wettbewerb und Subsidiarität. Wohlfahrtsverbände unterwegs in die Sozialwirtschaft. Berlin: Edition Sigma.

Deutscher Städtetag. (2010). Sozialleistungen der Städte in Not. Zahlen und Fakten zur Entwicklung kommunaler Sozialausgaben. Berlin: Deutscher Städtetag.

Grohs, S. (2007). Reform der Jugendhilfe zwischen Neuer Steuerung und Professionalisierung. Eine Bilanz nach 15 Jahren Modernisierungsdiskurs. Zeitschrift für Sozialreform, $53,247-274$.

Grohs, S. (2010). Modernisierung kommunaler Sozialpolitik. Anpassungsstrategien im Wohlfahrtskorporatismus. Wiesbaden: VS Verlag für Sozialwissenschaften.

Heinze, R. G., Schmid, J., \& Strünck, C. (1997). Zur politischen Ökonomie der sozialen Dienstleistungsproduktion. Der Wandel der Wohlfahrtsverbände und die Konjunkturen der Theoriebildung. Kölner Zeitschrift für Soziologie und Sozialpsychologie, 49, 242-271.

Holtkamp, L. (2010). Kommunale Haushaltspolitik bei leeren Kassen. Bestandsaufnahme, Konsolidierungsstrategien, Handlungsoptionen. Berlin: Edition Sigma.

Jann, W. (2005). Neues Steuerungsmodell. In B. Blanke, S. Bandemer, F. Nullmeier, \& G. Wewer (Hrsg.), Handbuch zur Verwaltungsreform (3. Aufl., S. 74-84). Wiesbaden: VS Verlag für Sozialwissenschaften.

Kommunale Gemeinschaftsstelle (KGSt). (1995). Das Neue Steuerungsmodell- Erste Zwischenbilanz. Bericht 10/1995. Köln: KGSt. 
Merchel, J. (1996). Neue Steuerung in der Jugendhilfe: handlungsfeldspezifische Differenzierungen im Kontext pluraler Trägerstrukturen, Teil 1. Nachrichtendienst des Deutschen Vereins für öffentliche und private Fürsorge, 213-220.

Merchel, J. (2003). Trägerstrukturen in der sozialen Arbeit. Eine Einführung. Weinheim: Juventa.

Merchel, J. (2006). $\$ 78$ b SGB VIII als Instrument zur Qualitätsentwicklung in der Erziehungshilfe? Ergebnisse einer Inhaltsanalyse von Qualitätsentwicklungsvereinbarungen. Zeitschrift für Kindschaftsrecht und Jugendhilfe, 78-89.

Merchel, J., \& Reismann, H. (2004). Der Jugendhilfeausschuss. Eine Untersuchung über seine fachliche und jugendhilfepolitische Bedeutung am Beispiel NRW. Weinheim: Juventa.

Münchmeier, R. (1995). Die Vergesellschaftung von Wertgemeinschaften: Zum Wandel der Jugendverbände in der Nachkriegs-Bundesrepublik. In T. Rauschenbach, C. Sachße, \& T. Olk (Hrsg.), Von der Wertgemeinschaft zum Dienstleistungsunternehmen. Jugend- und Wohlfahrtsverbände im Umbruch (S. 201-227). Frankfurt a. M.: Suhrkamp.

Münder, J., \& Ottenberg, P. (1999). Der Jugendhilfeausschuss. Münster: Votum.

Münder, J., Baltz, J., Kreft, D., Lakies, T., Meysen, T., Prosch, R., Schäfer, K., Schindler, G., Struck, N., Tammen, B., \& Trenczeck, T. (2006). Frankfurter Kommentar zum SGB VIII: Kinder- und Jugendhilfe (5. Aufl.). Weinheim: Juventa.

Parkinson, C. N. (1994). Parkinsons neues Gesetz. Reinbek bei Hamburg: Rowohlt.

Pluto, L., Gragert, N., Santen, E. van, \& Seckinger, M. (2007). Kinder- und Jugendhilfe im Wandel. Eine empirische Strukturanalyse. München: DJI.

Ronge, V. (1993). Die Verflechtung von Staat und Drittem Sektor auf kommunaler Ebene. In R. Voigt (Hrsg.), Abschied vom Staat-Rückkehr zum Staat? (S. 333-350). Baden-Baden: Nomos.

Seckinger, M., Weigel, N., \& Santen, E. van. (1998). Situation und Perspektiven der Jugendhilfe - eine empirische Zwischenbilanz. München: DJI.

Statistisches Bundesamt. (2011). Statistiken der Kinder-und Jugenhilfestatistiken. Ausgaben und Einnahmen. Wiesbaden: Statistisches Bundesamt.

Straub, U. (2004). Jugendhilfe im Spiegel der (Lokal-) Presse. Sozialmagazin, (4), 30-36. 